\title{
LA PERSPECTIVA DE LOS DOCENTES DE EDUCACIÓN PRIMARIA SOBRE LA REFORMA EDUCATIVA EN ZACATECAS, MÉXICO
}

\author{
ELEMENTARY SCHOOL TEACHERS PERSPECTIVE ABOUT EDUCATIVE REFORM IN \\ ZACATECAS, MÉXICO
}

\author{
Elizabeth Cristina Bañuelos-Sánchez ${ }^{1}$, Josefina Rodríguez-González ${ }^{(i D}$, Lizeth Rodríguez-González ${ }^{3}$ y Sergio \\ Aguilar-Reveles ${ }^{4}$ iD
}

\begin{abstract}
RESUMEN
En el presente artículo se describen los resultados de la investigación que fue realizada en el estado de Zacatecas, México, con el objetivo de conocer la percepción que tienen los docentes de educación primaria sobre la Reforma Educativa (RE) aprobada en el 2013. El marco teórico de la investigación son las reformas estructurales, que sientan las bases para analizar los cambios que reorganizan a las instituciones en conjunto. La recolección de la información se realizó mediante una entrevista con preguntas abiertas, enfocándose en los aspectos que tienen una repercusión directa sobre los docentes: la evaluación, los requisitos de ingreso y la permanencia. Los resultados dejan ver que los docentes en su mayoría no se oponen a la evaluación, mientras sirva como una herramienta para mejorar su práctica docente, aunque muestran desconfianza de la transparencia del sistema, al considerar que la RE es un mecanismo disfrazado para despedir maestros y que estos cambios no tendrán repercusiones en la calidad de la educación.
\end{abstract}

Palabras clave: Reforma Educativa; Educación primaria; Docentes; Evaluación.

\begin{abstract}
The results of a research carried out in the state of Zacatecas, México, aiming at probing teachers perceptions about the Educative Reform (RE) to elementary school education approved in 2013 in the country are presented. Structural reforms served as the research theoretical frame and are the starting point to analyze changes that reorganized educative institutions in general. The information gathering was conducted by an interview with open answers, focusing on the aspects which have direct effects on teachers: evaluation, entry requirements and the job continuity. The results show that most teachers do not oppose the evaluation, provided that it is used as a tool for improving their teaching practice. Yet they do mistrust the transparency of the system, since they consider that the RE is a hidden mechanism to fire teachers, instead of a mechanism to improve the quality of education.
\end{abstract}

Keywords: Educative Reform; basic education; teacher; evaluation.

Fecha de recepción: junio 16 de 2016 / Fecha de aceptación: marzo 31 de 2017 / Publicado en línea: abril 19 de 2017 Tipología: Artículo de Investigación Científica y Tecnológica

Para citar este artículo: Bañuelos, S. E., Rodríguez, G. J., Rodríguez, G. L. \& Aguilar, R. S. (2017). La perspectiva de los docentes de educación primaria sobre la reforma educativa en Zacatecas, México. Praxis, 13(1), 37 - 46. Doi: http://dx.doi.org/10.21676/23897856.2066

1. MCE. Universidad Autónoma de Zacatecas, México. Correo electrónico: eli cristy@hotmail.com ORCID: 0000-0003-1926-0550

2. Dra. Universidad Autónoma de Zacatecas, México. Correo electrónico: jrodrig22@hotmail.com ORCID: 0000-0002-6520-1343

3. Dra. Universidad Autónoma de Zacatecas, México. Correo electrónico: lizetharg@gmail.com ORCID: 0000-0002-6170-6918

4. Dr. Instituto Tecnológico Superior de Jerez, México. Correo electrónico: haciendact@yahoo.com.mx ORCID: 0000-0001-8397-5396 


\section{INTRODUCCIÓN}

$\mathrm{L}$ as reformas estructurales (RE) traen consigo un cambio simbólico, administrativo y laboral, entre otros aspectos, que modifica las condiciones de una organización, y se han concebido y construido a partir de la perspectiva de las economías dominantes, por lo que tienen un carácter político (Torres, 1997).

Las reformas estructurales al sistema educativo tienen como base las recomendaciones que emiten organismos Internacionales como la Organización para la Cooperación y el Desarrollo Económicos (OCDE), las cuales se sustentan en los resultados que obtienen los países miembros en las evaluaciones realizadas a los docentes y los alumnos. Entre sus objetivos está el avance educativo y económico de sus miembros (OCDE, 2014). Estas recomendaciones establecen el marco de las políticas públicas para implementar las RE a nivel mundial, buscando "reforzar las conexiones entre educación, empleo y el mejoramiento de la economía nacional, reducir los presupuestos educativos, establecer controles centrales más directos sobre el curriculum y la evaluación, y buscar mecanismos de elección y descentralización hacia las escuelas" (Gorostiaga \& Tello, 2011, p. 366).

En América Latina, desde los años 80, se han implementado diversas reformas estructurales, entre las que se encuentran las orientadas al sistema educativo. Las primeras RE estuvieron enfocadas en reformas hacia afuera, cuyo objetivo fue la ampliación de la cobertura de la enseñanza; la segunda generación en los años 90 fueron reformas hacia adentro; encaminadas a la calidad de la educación y la equidad, y que promovían políticas de cambios curriculares y prácticas pedagógicas, el establecimiento de sistemas de medición de la calidad (evaluación de rendimiento académico de los alumnos) e incentivos para los docentes con base en su desempeño (Guzmán, 2005). En la actualidad, la RE tiene un esquema desde arriba, donde:

Se asume que el cambio educativo puede lograrse no sólo "desde afuera" sino también "desde arriba" -típicamente, se habla de "bajar a la escuela", de "aterrizar en el aula"- mediante regulaciones, decretos, propuestas de nuevos planes y programas de estudio, etcétera. Se instala como natural la disociación entre quienes piensan, proponen, diseñan, dan, posibilitan, controlan, evalúan y encarnan el espíritu y en sentido mismo del cambio (arriba) y quienes se limitan a ejecutar y se someten a evaluación (abajo). La participación y la consulta social -a los docentes, los padres de familia, los alumnos, la sociedad en su conjunto- no tienen cabida ni razón de ser en este esquema. Todo cambio se piensa subsumido en la reforma. Incluso la innovación se piensa como patrimonio de la reforma, impedida o bien impulsada y legitimada desde arriba. Se cuestiona la 'pedagogía bancaria' en el aula, pero se mantiene en definitiva la "gran pedagogía bancaria” en el modelo mismo de reforma (Torres, 2000, p.9).

Este tipo de reformas se encuentran regularmente asociadas con las políticas generadas por los poderes políticos (Viñao, 2006) y los expertos en el tema, que fungen como diseñadores de políticas. Los cambios que se establecen en las reformas no siempre generan un avance en el sistema educativo, en muchas de las ocasiones pueden ser un retroceso en los logros. Según Osorio \& Pech (2007), solamente cuando la mayoría de los profesores se convierten en los agentes del cambio será posible promover una nueva cultura escolar y un cambio educativo.

Entre los factores que han contribuido al fracaso de las RE está su concepción de origen, que establece "el proceso como un secuencia lineal de evaluación, adopción e institucionalización donde los docentes son vistos como personas que responden pasivamente a los mandatos de los altos mandos burocráticos" (Datnow, Hubbard \& Mehan, 1998, p.2), y deja de lado el papel activo y el rol que juegan los docentes como agentes que pueden actuar de distinta manera ya sea para impulsar los cambios, para resistirlos o subvertirlos.

La implementación de una reforma implica un contraste entre los diseñadores de políticas y los docentes, lo que produce conflictos entre los maestros que las consideran imposiciones externas a su contexto escolar, que dejan de lado sus opiniones, sentir y pensar. Esta percepción provoca un caos interno que les genera 
preocupaciones ante la imposiciones externas que chocan con las expectativas que ellos mismos van formando. Esta intensificación laboral "puede desembocar en la pérdida de competencia profesional, cambios en la concepción de las tareas y una ética profesional diferente” (López \& Tinajero, 2009, p. 1195).

La perspectiva de los docentes se forma por el cúmulo de creencias, actitudes y significados, con base en su experiencia escolar, personal y el conocimiento. Según Viñao (2006), las RE "producen inquietud y desasosiego en los profesores, sobre todo en aquellos que puedan ver modificado el lugar, el tipo y las condiciones de trabajo" (p.51). Autores como Viñao (2006) y Datnow, Hubbard \& Mehan (1998) consideran que las RE fracasan porque no toman en cuenta a los actores centrales del proceso "los docentes", debido a que:

Un aspecto central lo constituye la identificación de los aspectos de las reformas que más les preocupan. La ambigüedad sobre las nuevas tareas que deben realizar, el número y frecuencia de los cambios impuestos en poco tiempo, y la incertidumbre de poder cubrir las demandas, son algunos de los elementos que generan inquietud y afectan la identidad de los docentes. Además los programas de capacitación constituyen muchas veces esfuerzos fragmentados que carecen de oportunidades reales para que los maestros pongan en práctica los cambios esperados (Van den Berg, 2002, citado por López \& Tinajero, p. 1196).

La forma en que haya sido implementada la reforma será fundamental para lograr los objetivos planteados, pues el docente es un actor central en la transformación y el cambio del sistema educativo, ya que es quien conoce y vive la realidad de las escuelas.

\section{Características de la Reforma Educativa en México}

En el año 2013 se aprobó la propuesta del presidente Enrique Peña Nieto, sobre una nueva reforma constitucional en materia educativa. El objetivo de la RE se centra en la necesidad de mejorar la calidad y equidad en la educación (Presidencia de la República, 2013). La Reforma
Educativa se enfocó en la educación básica, que integra los niveles de Preescolar, Primaria y Secundaria. La Educación Primaria comprende el segundo nivel educativo; en este trayecto de seis grados, se da continuidad a las competencias de la Educación Preescolar; el nivel de primaria tiene como objetivo que el alumno adquiera los conocimiento básicos en matemáticas, español, formación cívica y ética, geografía, historia y ciencias naturales, lo que establece las bases para que el alumno logre el perfil de egreso para la Educación Secundaria (SEP, s/f).

La reforma a la constitución y las leyes secundarias son el punto de partida que constituye la base jurídica para dar curso a la creación de nuevas condiciones laborales y administrativas. Se pretende que la RE sea progresiva y mejorar con ella las prácticas educativas y el aprendizaje de los alumnos. Con la RE se promulgan la Ley General del Servicio Profesional Docente (DOF, 2013), la Ley del Instituto Nacional para la Evaluación de la Educación (DOF, 2013b); además, se adicionan reformas a la Ley General de Educación (DOF, 2013a) y a la Constitución Política de los Estados Unidos Mexicanos (DOF, 2014).

Las modificaciones al artículo tercero constitucional establecen la calidad en la educación, la evaluación obligatoria, los concursos de oposición para ingresos, promociones, reconocimientos y permanencia (López, 2013), como los nuevos lineamientos a los que deberán sujetarse los maestros ante la autonomía vigente del Instituto Nacional para la Evaluación de la Educación (INEE) mediante el Sistema Nacional de Evaluación Educativa (SNEE).

El INEE será el responsable de diseñar los lineamientos generales de evaluación a los que se sujetarán las autoridades educativas, así como de evaluar la calidad, el desempeño y los resultados del Sistema Educativo Nacional a la educación básica y a la educación media superior; es un organismo público autónomo, que posee personalidad jurídica y patrimonio propios, tiene plena autonomía técnica de gestión, presupuestaria y de organización interna (DOF, 2013b). El INEE coordina el Sistema Nacional de Evaluación Educativa (SNEE) y tienen como 
atribuciones definir los procesos de evaluación a los docentes, para el ingreso al servicio docente y la promoción, entre otros.

En el artículo 73, fracción XXV de la Constitución, se agrega el Servicio Profesional Docente (SPD), que es regulado por la Ley General del Servicio Profesional Docente, encargada de regir y establecer los criterios, los términos y condiciones para el ingreso, la promoción, el reconocimiento y la permanencia en el servicio de los docentes. Su objetivo es regular el SPD en la educación básica y media superior, establecer los perfiles, parámetros e indicadores, regular los derechos y obligaciones derivados y, asegurar la transparencia y rendición de cuentas (DOF, 2013).

Dentro de los artículos transitorios se incluye el proceso para integrar la junta de gobierno del INEE, la evaluación del desempeño docente que integra la formación, actualización, capacitación y superación profesional; así mismo, se integra la autonomía de gestión para las escuelas y la continuación con las escuelas de tiempo completo (López, 2013).

En la Ley General de Educación se establece la obligación de las autoridades educativas, federales y locales, de poner en marcha lo necesario para revisar el modelo educativo en su conjunto, los planes, programas, materiales y métodos educativos; esta ley se encarga de regular el SNEE (DOF, 2013a). En la RE se plantea que los maestros serán seleccionados para el servicio público educativo, con base en el mérito y en sus capacidades. Textualmente se señala que:

Los procesos de evaluación de los docentes permitirán saber cuáles son sus necesidades de regularización y formación continua, para mejorar sus capacidades... ningún docente con nombramiento definitivo, a la entrada en vigor de la ley y que cumpla será afectado en su derecho al empleo, además otorgará gratuitamente programas de regularización y formación continua a los maestros para mejorar sus capacidades, además plantea un plan integral para impulsar, rediseñar y fortalecer a la educación normal (SEP, 2015, p.1).

Como todo proceso de cambio, la RE genera expectativas en los actores involucrados. Para el caso de México, se han dado principalmente en los docentes, por las implicaciones que tiene la reforma en aspectos laborales, de gestión y académicos. Partiendo de esta premisa se hace el siguiente planteamiento del problema.

\section{Planteamiento del problema}

Ante la aprobación de la RE en México que el gobierno de Enrique Peña Nieto propone para la educación básica, como una continuación de las políticas de los gobiernos anteriores y las recomendaciones emitidas por organismos internacionales, las inconformidades de los docentes no se hicieron esperar; muchos de ellos mostraron su desacuerdo (por las implicaciones que tiene la reforma hacia los maestros) realizando protestas en la capital del país y en diferentes estados, con marchas, cierre de escuelas y tomas de autopistas, entre otras acciones, para hacer ver al gobierno y a las autoridades educativas su oposición a la RE.

En el estado de Zacatecas el escenario fue similar, los docentes de distintas escuelas se unieron a los actos de protesta que se realizaron a nivel nacional, participando en la marcha nacional que convocó la Coordinadora Nacional de Trabajadores de la Educación (CNTE); además, se hicieron tomas de la Secretaría de Educación de Zacatecas (SEDUZAC) y Ciudad Gobierno por varios días, lo que impidió las actividades normales en estas instalaciones. Se destaca que un grupo de maestros entraron a la televisora Televisa para pedir que los dejaran hablar en televisión abierta. A las manifestaciones se integraron padres de familia que marcharon conjuntamente con los maestros y tomaron escuelas, con el propósito principal de lograr la abrogación de la reforma (Amador, 2013).

Analizando los hechos ocurridos, las manifestaciones realizadas, las inconformidades y el rechazo mostrado por los docentes ante la $\mathrm{RE}$, surgen una serie de inquietudes que sirvieron como punto de partida para la presente investigación, teniendo en cuenta que uno de los principales actores de este proceso son los docentes en quienes la RE tendrá una mayor incidencia. En este sentido, se estableció como 
objetivo general conocer la percepción de los docentes de educación primaria del estado de Zacatecas sobre la Reforma Educativa, enfocándose en los aspectos que tienen una repercusión directa sobre los maestros: la evaluación, los requisitos de ingreso y la permanencia.

\section{METODOLOGÍA}

La investigación fue de tipo exploratoria y de corte cualitativo, se enfocó en la educación primaria debido a que este nivel concentra el grueso de la población docente y alumnos inscritos en el sistema educativo mexicano. La recolección de la información se realizó mediante un cuestionario de preguntas abiertas divididas en dos bloques: el primero enfocado en conocer los datos personales del informante y sus características laborales; el segundo sobre la RE, profundizando en la evaluación al desempeño docente y la promoción, así como, el ingreso y la permanencia.

Para la selección de los informantes se establecieron las siguientes características: en primer lugar, integrar docentes de educación primaria que laboraran tanto en la zona urbana como en la rural. Contar con ambos escenarios permitió tener una perspectiva más amplia sobre los contrastes y visiones que la RE genera en los docentes que tienen condiciones de trabajo muy distintas. Este aspecto tomó importancia en la investigación debido a que en el estado de Zacatecas, muchas de las escuelas en la zona rural presentan carencias importantes, entre las que se encuentran la falta de servicios básicos, como agua y luz, otras más no cuentan con acceso a internet e incluso sus instalaciones no son suficientes, de modo que el profesor tiene que trabajar en salones improvisados, lo que contrasta con las condiciones que tienen los docentes de escuelas urbanas que cuentan en su mayoría con todos los servicios.

En segundo lugar, considerando que la permanencia es otro de los factores que modifica la RE, se incluyó en la investigación tanto maestros de base como de contrato, para tener la perspectiva de los docentes que tienen diferentes condiciones laborales. Finalmente, se buscó integrar a hombres y mujeres con la intención de que la muestra fuera equitativa.

Para la aplicación del cuestionario se realizó la invitación abierta y de forma voluntaria. A un grupo de docentes se les aplicó de manera personal en formato impreso, y se respondió en ese momento cualquier duda que tuvieran con respecto al instrumento o a las preguntas. A otro grupo de docentes se les envió el cuestionario en formato digital mediante correo electrónico o por la red social Facebook, lo que ayudó para recabar un mayor número de entrevistas, debido a que muchos de los docentes se mostraron renuentes y a la defensiva: algunos, arguían que tenían prohibido dar opinión pública con respecto a la $\mathrm{RE}$, otros expresaban sentir temor de que se pudiera filtrar la información y ser afectados en su trabajo; esta estrategia también sirvió para que los encuestados se sintieran con mayor confianza y respondieran abiertamente a las preguntas. Así mismo, se dio la oportunidad a los docentes que no desearan dar su nombre de que pusieran un seudónimo y con ello guardar la confidencialidad del informante.

El cuestionario se aplicó entre los meses de junio-agosto del 2014, poco tiempo después de ser aprobada la RE y antes de que se aplicaran las primeras evaluaciones a los docentes; fue entonces un primer acercamiento al sentir y pensar del docente sobre la RE. El total de maestros que colaboraron en la investigación fueron 20; de estos, 12 correspondieron a hombres, nueve de base y tres de contrato, seis trabajan en zona rural, cinco en zona urbana y uno no contestó a este renglón; ocho fueron mujeres, de las cuales tres fueron de base y cinco de contrato, cinco trabajan en zona urbana y tres en zona rural. Los docentes que contestaron el cuestionario laboran en los municipios de Jerez, Zacatecas, Loreto, Noria de Ángeles, Fresnillo, Villanueva, Calera de Víctor Rosales y San Pedro Piedra Gorda.

Una vez aplicados los cuestionarios, se procedió a transcribirlos y ordenar la información obtenida en un formato claro y de fácil interpretación, utilizando una hoja de cálculo de Excel de la paquetería Microsoft Office. Se establecieron cinco 
categorías de análisis para el procesamiento de la información: 1) datos personales, 2) datos laborales, 3) RE, 4) evaluación al desempeño y la promoción y 5) permanencia. Las categorías 1 y 2 estuvieron enfocadas en conocer los datos del informante, las categorías 3, 4 y 5 se orientaron a indagar sobre la precepción y conocimiento que tienen los docentes del tema; en los resultados se integraron los porcentajes según las opiniones encontradas, lo que permitió conocer las tendencias de las opiniones y percepciones de los encuestados.

\section{RESULTADOS Y DISCUSIÓN}

La opinión que los docentes entrevistados tienen de la RE en su mayoría es negativa, debido a que la asocian al despido y control de los maestros. Consideran que es un modelo político-económico que no encaja con las necesidades del sistema educativo mexicano, que mide a todos por igual, cuando las condiciones que tienen un maestro en las escuelas urbanas es por mucho más ventajosa que la de quienes trabajan en la escuela rural. Además mencionan que será difícil implementarla y llevarla a cabalidad, debido a que existe corrupción dentro del sistema educativo, por lo que la reforma debería ser desde arriba para después comenzar con los maestros.

Entre los aspectos positivos que los docentes consideran que generará la RE está la autonomía de gestión que tendrán las escuelas, debido a que recibirán apoyos económicos anualmente. El 55 $\%$ de los docentes encuestados consideran que si es implementada de forma correcta, segura e imparcial la RE generará beneficios como la posibilidad de capacitación y actualización, y la de conocer sus debilidades y tener la oportunidad de combatirlas. Los maestros de contrato y con perfil profesional diferente al normalista piensan que les abre una oportunidad para obtener un lugar definitivo en el mundo laboral escolar.

El $95 \%$ de los docentes expresan que la RE viola sus derechos laborales, pues les condiciona la plaza al resultado que obtengan en la evaluación, por lo que no existirá estabilidad laboral, igualdad y beneficios como trabajadores, tampoco tendrán un sindicato que los defienda; les dará el nombre de incompetentes cuando no aprueben, y los hará acreedores al despido sin tener oportunidad de defenderse, lo que no sólo afectará al docente, sino también la estabilidad del alumno.

Con respecto a si la RE mejorará sus condiciones de trabajo, los docentes consideran que no habrá cambios, debido a que tanto la infraestructura como el ambiente escolar seguirán siendo los mismos. Según su opinión, la reforma se enfoca principalmente en el docente, dejando de lado el salón de clases y el entorno donde se desenvuelve el alumno, no se toman en cuenta los problemas familiares, económicos, de salud, entre otros factores que afectan el desempeño académico de los estudiantes. Los docentes encuestados consideran que para lograr un verdadero cambio en la educación primaria debe ponerse atención en mejorar la infraestructura de las escuelas, principalmente las que se encuentran en las zonas rurales; en combatir la corrupción que existe en el sistema educativo, por ejemplo por el desvío de recursos; además que el gobierno se involucre de forma adecuada ayudando a mejorar el entorno del alumno. El docente J.M. adscrito a la primaria Soledad Fernández, ubicada en la ciudad de Zacatecas, afirma que:
Para mejorar la educación en México, el proble- ma no es sólo los maestros, creo que existe un problema más de fondo y son los altos mandos, la desviación de los recursos, la infraestructura y la desigualdad que existe en las escuelas y la reforma sólo se enfoca en los maestros, pero para que un alumno aprenda hay que cambiar todo su entorno, empezando desde casa. Se debe refor- mar todo el sistema educativo y que el gobierno se involucre de la forma adecuada, no sólo para querer atacar al maestro y echarle la culpa a él.

El $45 \%$ de los docentes encuestados no confían en la evaluación que plantea la RE, dudan de la transparencia y la legalidad de los resultados, consideran que no tienen garantías que los respalden, además de verlo como un instrumento lineal que mide a todos por igual, sin tener en cuenta las condiciones de trabajo a las que se 
enfrentan. En este sentido, el docente por contrato L.H. de la Primaria Ramón López Velarde en la ciudad de Jerez manifiesta:

va [a] perjudicar a todos, porque nos evaluarán a todos los maestros de forma general, cuando las condiciones que tienen no son las mismas, no es igual trabajar en una comunidad [zona rural] que en la cabecera municipal [zona urbana]. Los alumnos que estudian en la comunidad a veces ni agua, luz o bancas dignas tienen en sus salones a comparación de los que están en la cabecera.

Con la RE se crea el INEE como organismo encargado de desarrollar, realizar e implementar el examen de evaluación a los docentes. El total de docentes encuestados dicen conocer el organismo. Sin embargo, solo el 90 \% sabe cuáles son sus atribuciones, lo definen como el organismo que se encarga de la evaluación, la promoción y quien determina la situación de los docentes dentro del sistema educativo. Algunos docentes son más tajantes y consideran que se encarga de clasificar a los docentes como incompetentes con base en evaluaciones radicales, además de tener la facultad de despedirlos.

El $80 \%$ de los docentes encuestados conocen los mecanismos e implicaciones de la evaluación que realizará el INEE, coinciden en que se les aplicará un examen de manera general a todos los maestros donde se evaluará la planeación, los contenidos, los resultados obtenidos por sus alumnos, el trabajo y el espacio escolar; tendrán tres oportunidades para aprobarlo, aunque no tienen claro cómo se llevará a cabo.

Los docentes desconfían del INEE y de la trasparencia del proceso. El $74 \%$ no creen que los procesos se den de manera clara y afirman que esto difícilmente se puede lograr, debido a la corrupción que existe, pues en las evaluaciones anteriores que han realizado no se les han mostrado sus resultados, además de que existen muchos intereses de por medio. Sin embargo, el 85 \% manifestó estar de acuerdo en ser evaluados, siempre y cuando el examen sea confiable y sirva como un punto de apoyo en su desempeño, tenga una retroalimentación para conocer cuáles son las áreas que necesitan reforzar y capacitarse para mejorar su práctica docente, considerando que el examen solo calificará el conocimiento que tienen el docente, dejando de lado sus habilidades y aptitudes desarrolladas en el salón de clases. O como lo sostiene uno de los encuestados:

Yo sí estoy de acuerdo en que nos evalúen, pero que la evaluación sea confiable, y que nos muestren los resultados, porque así, uno puede ver en que anda mal y echarle ganas. Las evaluaciones siempre son buenas, pero ésta la quieren utilizar para destituir al maestro o para darnos una mala imagen, de que estamos reprobados (docente por contrato J.P. de la escuela Primaria Olimpiada en Calera de Víctor Rosales, Zacatecas).

El mismo porcentaje ( $85 \%$ ) está de acuerdo en ser evaluado periódicamente mientras no sean violentados sus derechos, ni se empleen los resultados como un justificante para removerlos de su puesto o despedirlos del empleo. Respecto a los parámetros de evaluación, contestaron que no son adecuados, porque evalúan a los docentes de forma general, cuando las condiciones que enfrentan en sus aulas son muy diferentes, por lo que no creen que la evaluación permanente ayude a mejorar el desempeño docente, ya que si fuera éste el objetivo existirían cursos antes y después de la aplicación de los exámenes.

A pesar de lo anterior, el $55 \%$ considera que la evaluación les permitirá conocer cuáles son sus necesidades de regularización y formación continua para mejorar su práctica docente, siempre y cuando se realice de manera correcta y se dé seguimiento, pero dudan que esto suceda, consideran que es una estrategia del gobierno para justificar el despido de maestros y el cierre de escuelas. El resto, el $45 \%$, no cree que los procesos de evaluación permitan conocer cuáles son sus necesidades de regulación, ya que para ellos, en el examen solo se califica el conocimiento del docente y no la forma en que se desempeñan en el aula.

Sobre los incentivos económicos que plantea la RE para los docentes conforme a los resultados que obtengan en las evaluaciones, el 75 $\%$ considera que sería un buen aliciente para seguirse preparando. Con respecto a que la promoción se realice por examen de oposición, el 
90 \% están de acuerdo, considerando que con este mecanismo será más fácil acceder a los puestos directivos y será una oportunidad para las personas que tienen la capacidad de estar al frente de una escuela con un mejor salario. Sin embargo, algunos lo consideran como un ideal, porque creen que es difícil que esto suceda, por el tráfico de influencias que existe, por los intereses de grupos y por la corrupción que hay en el sistema educativo. Tal como lo manifiesta el docente J.H., quien ha laborado en la zona rural y que al momento de la entrevista se encontraba en espera de contrato:

Yo estoy de acuerdo, porque esos cargos se los dan a los empleados de confianza o a los que tienen un amigo en los altos mandos y muchas veces no es gente que esté muy capacitada. Donde he trabajado los directores son los que menos hacen cosas y los que ganan más, eso es ilógico.

La opinión que tienen los docentes sobre los requisitos de ingreso y permanencia que establece la RE es la siguiente: el $95 \%$ manifiesta estar de acuerdo en que la obtención de plazas sea por concurso, debido a que antes de la reforma existían diferentes mecanismos para la asignación de una plaza de base en la educación primaria, los cuales iban desde un examen de oposición, por convenios con las normales, hasta las plazas heredadas. Los docentes por contrato consideran que la RE abre una oportunidad para obtener un lugar definitivo, con base en sus méritos, sin importar la carrera que tengan.

La mayoría de los entrevistados (80 \%) coinciden en que los requisitos de ingreso y de obtención de plaza sean por examen de oposición. En lo que no están de acuerdo es en que las plazas se abran a todos los profesionistas independientemente de la carrera que tengan; consideran que deben ser los normalistas o estudiantes con perfil docente los únicos que puedan participar, ya que fueron formados para ser maestros conforme al currículo aprobado por el gobierno. Al respecto, el docente A.B., maestro de educación física en la escuela Vicente Guerrero, en la Quemada, perteneciente al municipio de Villanueva, Zacatecas, comenta:
Estoy en total desacuerdo ya que no cualquier profesionista tiene los principios pedagógicos que los maestros normalistas tenemos, además de que los normalistas o egresados de escuelas similares que tienen la carrera de educación, tienen el perfil que el mismo gobierno [pide]a través de los planes de estudio y la formación magisterial de estas escuelas que son las que ponen en práctica para los aspirantes a las diferentes licenciaturas ligadas a la educación básica y no es justo que el mismo gobierno que pide esos planes, sea el que ahora le dé puerta abierta a los otros profesionistas.

Respecto a la percepción que tienen los docentes entrevistados sobre los mecanismos para la permanencia, el $85 \%$ considera que estos lineamientos afectarán sus derechos laborales, su estabilidad económica y su calidad de vida, lo que dejará al maestro desarmado para defenderse, debido a que en este esquema, el sindicato no tendrá facultades para proteger a sus agremiados; además, sienten que quedarán estigmatizados socialmente. Así lo manifiesta el docente M. R. de la escuela Primaria Benito Juárez García, en Jerez, Zacatecas:

Sí, porque no te dan opciones para permanecer en tu trabajo, solo se basan en un examen, si sales mal, vas para afuera y no puedes hacer nada, además, ahora el sindicato ya no te podrá defender porque también lo quieren desaparecer, entonces si no tienes quién te apoye por más que pelees, si te quieren correr del empleo no podrás hacer nada.

Según lo que establece la RE, los docentes de base que no aprueben el examen en las tres oportunidades a que tienen derecho, cambiarán su figura a funciones de apoyo. El $95 \%$ de los encuestados expresa no estar de acuerdo, opina que es injusto, debido a que un examen no engloba todo el desempeño del docente, y que así se minimiza su papel. Otros consideran que con esta medida el sistema educativo integrará a jóvenes inexpertos que aceptarán las condiciones que les impongan con tal de tener un trabajo. Los docentes que no cuentan con un contrato de base lo ven como una oportunidad para obtener un lugar definitivo, y para concursar en iguales condiciones con otros profesionales. 


\section{CONCLUSIONES}

La investigación realizada tuvo un carácter exploratorio y descriptivo, fue aplicada meses después de la aprobación de la RE, cuando las condiciones de la evaluación aún no estaban establecidas de manera definitiva. Se puede observar que las opiniones de los encuestados coinciden con la teoría, que asumen la RE como un proceso desde afuera, una imposición que daña sus derechos laborales, lo que los mantiene a la expectativa de los giros que puedan perjudicar su empleo; sienten la RE como un atentado a los derechos ya adquiridos en las conquistas obtenidas dentro del contrato colectivo; la ven no como una retroalimentación, sino como una persecución.

Los maestros normalistas encuestados manifiestan que la RE es una “invasión” laboral de otros profesionistas al ramo de la educación básica, y que fomenta una competencia desleal en contra de los que se han formado para ser maestros, punto de vista que no comparten los profesionales de otras áreas.

Desde la óptica de los encuestados, el uso de una herramienta tradicional como el examen de opción múltiple no abastecerá una calidad educativa, sino que, al contrario, recaerá en el estado anímico del maestro al sentirse vulnerable ante la sociedad (padres de familia y alumnos) y al poner en jaque su empleo. Coinciden en que evaluación no es sinónimo de examen, dudan de contestar sobre quién evaluará a los evaluadores, cuál será el futuro de la figura y el rol del docente dentro del sistema educativo.

Los resultados de reformas anteriores muestran que los cambios no se han reflejado en la mejora de los indicadores; el escenario ante esta reforma según los docentes encuestados no tendrán una repercusión educativa en los alumnos, debido a que no considera el entorno de la escuela, de los alumnos, ni maestros, es más un mecanismo de control de los docentes mediante evaluaciones.

Este primer acercamiento, sin duda, deja abiertos caminos para el análisis en futuras investigaciones y deja preguntas abiertas para seguir explorando sobre la RE, para contrastar cómo se ha modificado la percepción de los docentes sobre la RE después de haber sido evaluados, cuáles son las opiniones de los docentes que no aprobaron la evaluación, qué opinan los maestros que han sido removidos de sus cargos, qué piensan los docentes que lograron una plaza, además de indagar si se han logrado los cambios educativos planteados.

\section{REFERENCIAS BIBLIOGRÁFICAS}

Amador, J. (2013). 15 mil maestros marchan por centro histórico de Zacatecas. Recuperado de: http://zacatecasonline.com.mx/noticias/ local/34354-15-mil-maestros-marchan.html

Datnow, A., Hubbard, L., \& Mehan, H. (1998). Educational reform implementation: A co-constructed process, Research Report núm. 5, Santa Cruz, California: Center for Research on Education, Diversity \& Excellence.

Diario Oficial de la Federación (DOF) (2013). Decreto por la que se expide la Ley General del Servicio Profesional Docente: Presidencia de la República. México.

(2013a). Decreto por el que se reforman y adicionan y derogan diversas disposiciones de la Ley General de Educación. Poder Ejecutivo y Secretaría de Educación Pública. México.

(2013b). Decreto por el que se expide la Ley del Instituto Nacional para la Evaluación de la Educación . Presidencia de la República. México. (2014c). Decreto por el que se reforman, adicionan y derogan diversas disposiciones de la Constitución Política de los Estados Unidos Mexicanos, en Materia políticoelectoral. Poder Ejecutivo y Secretaría de Gobernación. México.

Guzmán, V. C. (2005). Reformas educativas en América Latina: un análisis crítico. Revista Iberoamericana de Educación, 36(08).

Gorostiaga, J. M., \& Tello, C. G. (2011). Globalización y reforma educativa en América Latina: un análisis inter-textual. Revista Brasileira de Educação, 16(47), 363-388.

López, B. G., \& Tinajero V. G. (2009). Los docentes ante la reforma del bachillerato. Revista Mexicana de Investigación Educativa, 14, 1191-1218. 
López, A. M. (2013). Una reforma "educativa” contra los maestros y el derecho a la educación. El Cotidiano, 179, 55-76.

Organización para la Cooperación y el Desarrollo Económicos (OCDE) (2014). El trabajo de la OCDE. Recuperado de: http://www.oecd.org/ centrodemexico/laocde/eltrabajodelaocde.htm

Osorio, P. R., \& Pech, C. S. (2007). Preocupaciones de los profesores ante la reforma integral de la educación secundaria en México REICE. Revista Iberoamericana sobre Calidad, Eficacia y Cambio en Educación, 5(3), 173-189.

Presidencia dela República (2013). ReformaEducativa. Recuperado de: http://www.presidencia.gob.mx/ reformaeducativa/\#leyes-secundarias

Secretaría de Educación Pública (SEP) (2015). Principios relevantes de la reforma educativa. Recuperado de: http://www.sep.gob.mx/ work/appsite/cuarto_de_plana.pdf
Secretaría de Educación Pública (SEP) (s/f). Programas de Estudio Primaria. Recuperado de http://www.curriculobasica.sep.gob.mx/ index.php/prog-primaria

Torres, J. C. (1997). Las dimensiones políticas e institucionales de las reformas estructurales en América Latina. Naciones Unidas. Recuperado de: http://repositorio. cepal.org/bitstream/handle/11362/7369/ S9700017_es.pdf?sequence $=1$

Torres, R. M. (2000). Reformas Educativas, docentes y organizaciones docentes en América Latina y el Caribe En: Los docentes protagonistas del cambio educativo. Bogotá: CAB/ Editorial Magisterio Nacional.

Viñao, A. (2006). El éxito o fracaso de las reformas educativas: condicionantes, limitaciones, posibilidades. En Morata (Ed). La reforma necesaria: entre la política educativa y la práctica escolar, (pp. 43-60), Madrid. 